TF-CBT and EMDR for young people with trauma and psychosis (using a phasic treatment approach) Two Early Intervention Service Case Studies

\begin{tabular}{|r|l|}
\hline Journal: & The Cognitive Behaviour Therapist \\
\hline Manuscript ID & Draft \\
\hline Manuscript Type: & Practice Article \\
\hline Keywords: & PTSD, psychosis, therapy, traumatic stress, CBT \\
\hline
\end{tabular}

SCHOLARONE ${ }^{\text {M }}$

Manuscripts 


\title{
TF-CBT and EMDR for young people with trauma and psychosis (using a phasic treatment approach) Two Early Intervention Service Case Studies
}

\begin{abstract}
Background

The relationship between trauma and psychosis is well established with a large amount of overlap between the ICD/DSM diagnostic criteria for Post-traumatic Stress Disorder (PTSD) and psychosis and/or schizophrenia. In spite of co-morbidity and evidence of the links and underlying mechanisms, trauma is rarely a focus of intervention in psychosis. Psychosis has often been on the list of exclusion criteria for PTSD research studies. There is a lack of literature on the impact of trauma work with people experiencing psychosis. The National Institute for Health and Care Excellence (NICE)(2014) suggest that Early Intervention in Psychosis (EIP) service users should be assessed for PTSD and PTSD guidelines (NICE, 2005) followed for those who show who show signs of post-traumatic stress.
\end{abstract}

\begin{abstract}
Aims
There is a need to evaluate the effectiveness of therapeutic approaches for people with PTSD and co-morbid psychosis. These case studies aim to provide initial evidence of how two EIP clients experienced and responded to NICE-recommended psychological therapy for trauma.
\end{abstract}

\section{Method}

Two EIP clients received psychological therapy (including trauma-focused Cognitive Behavioural Therapy (CBT) and Eye Movement Desensitisation and Reprocessing (EMDR)) for identified traumatic experiences. Assessment outcome measures were utilised to establish the effectiveness of the interventions. 
TFCBT and EMDR for young people with trauma and psychosis

\section{Results}

Both clients reported significant improvements following therapy, including reduced trauma-related distress, reduced distress from symptoms of psychosis and improved quality of life.

\section{Conclusions}

Clients with co-morbid PTSD and symptoms of psychosis are likely to benefit from recommended psychological treatments for PTSD. Further research is required to address generalisability to a larger population.

Keywords: Trauma, PTSD, Psychosis, EMDR, TF-CBT, Early Intervention 
TFCBT and EMDR for young people with trauma and psychosis

\section{Introduction}

The relationship between trauma and psychosis is well established. This includes evidence of a doserelated-response to trauma as well as certain childhood adversities being associated with specific symptoms (Morrison, Frame \& Larkin, 2003, 2006; Bentall, Wickham, Shevlin \& Varese, 2012; Varese et al, 2012). Alongside this, auditory hallucinations are common in people with posttraumatic stress disorder (PTSD) (Thomas et. al, 2014). Mechanisms linking the two concepts have been proposed and investigated, such as: Attachment and emotional resilience (Sitko, Bentall, Shevlin, O'Sullivan \& Sellwood, 2014), the traumatogenic neurodevelopmental model (Read et al 2001), the interaction between information-processing and stress (catastrophic interaction hypothesis) and Morrison's integrated cognitive model (Morrison, Frame \& Larkin, 2003) which frames hallucinations as decontextualized flashbacks and unusual beliefs as attempts to explain trauma-related hallucinations (linked to beliefs about themselves, others and the world).

There is large amount of overlap between the ICD/DSM diagnostic criteria for PTSD and psychosis and/or schizophrenia. In spite of the level of co-morbidity and the evidence for the links and the underlying mechanisms, trauma is rarely a focus of intervention in psychosis. Service users with psychosis are not routinely screened for PTSD and treatments have historically focused on the symptoms of psychosis. Trauma-focused Cognitive Behaviour Therapy (CBT) and Eye Movement Desensitisation and Reprocessing (EMDR) are recommended as frontline treatments for PTSD and yet therapists do not routinely offer them to service users with symptoms of psychosis and PTSD. For example, results that found that $33 \%$ of a sample of patients with a severe mental illness within a community mental health setting met the diagnostic criteria for PTSD, though only $4 \%$ of the sample had a formal diagnosis of PTSD in their medical record (Howgego et al 2005).

Alongside this, psychosis has often been on the list of exclusion criteria for research studies on PTSD. As a result, there is a lack of published literature on the impact of trauma work on PTSD and psychosis. 
TFCBT and EMDR for young people with trauma and psychosis

One randomised controlled trial has examined the use of cognitive restructuring with service users with severe mental illness and found a modest positive effect. However, only $16 \%$ of the sample had schizophrenia or schizoaffective disorder (Watts et al., 2013). Two pilot studies, one examining the efficacy of Prolonged Exposure (PE) and the other EMDR found large effects on PTSD (Frueh et al., 2009; Van den Berg \& Van der Gaag, 2012) as did a controlled case series study examining both approaches (de Bont, van Minnen \& de Jongh, 2013).

There has been one multi-site randomised controlled trial comparing the effects of PE, EMDR and waiting list on service users with a diagnosis of psychosis and co morbid PTSD (de Bont, van den Berg, \& van der Vleugel, 2013; Van den Berg, et al, 2015). Participants in the PE and EMDR conditions showed a greater reduction of PTSD symptoms compared to those in the waiting list group; these participants were also more likely to subsequently not meet criteria for a diagnosis of PTSD a difference maintained at 6 month follow-up. Both standard PE and EMDR protocols were found to be safe and effective with this client group. This study focused on participants with longstanding psychotic symptoms and a clear diagnosis of both PTSD and schizophrenia.

Early intervention services in the UK were initially provided to care for young people aged between 14 and 35 with a first episode of psychosis in the community EIP Services (EIPS) in the UK (Policy Implementation Guide, 2003) but the service has now been extended to 14 to 65 year olds (NICE, 2016). Recent NICE Guidelines for Psychosis and Schizophrenia (2014) recommend the use of CBT for Psychosis and suggest the need to assess for PTSD and then to follow PTSD NICE guidelines (2005) for those who show signs of post-traumatic stress. There has been no published research examining the safety and efficacy of interventions for post-traumatic stress within an early intervention population and without a formal diagnosis of PTSD. This study seeks to address this.

The Lancashire Traumatic Stress Service (LTSS) was set up as a tertiary level out-patient service to provide specialist psychological and pharmacological interventions for individuals presenting with symptoms of post-traumatic stress disorder within secondary care mental health services. Service 
TFCBT and EMDR for young people with trauma and psychosis

users referred to the service typically presented with multiple traumatic experiences often commencing in childhood. The service provides a 'phasic' or 'modular' approach. This prepares service users to manage the demands of trauma-focused interventions and offer therapists confidence in facilitating such interventions. Phasic or modular treatment has been found to be effective with clients with complex presentations (Cloitre et al, 2011).

The literature on working with trauma emphasise the importance of clinician self-care and managing the risks of potential burnout due to prolonged exposure to vicarious trauma (Baird \& Kracen, 2006). The structure within the Lancashire Traumatic Stress Service emphasises regular case discussion via a weekly team supervision approach to ensure the work is contained and the team's clinical expertise is available for the benefit of all service users.

This study was designed to evaluate the impact of providing trauma-focused psychological interventions to service users presenting to an early intervention in psychosis service who also had a history of traumatic experiences.

The aims were three-fold:

1. Does the LTSS modular approach translate appropriately to young people with psychosis (i.e. to ensure that the interventions within the modular approach could be tailored to both service users' priorities and goals, and evidence based practice)?

2. Do the safety and stabilisation interventions (initial phases of modular approach) prepare service users to manage the demands of exposure-based interventions (second-line interventions: PE; EMDR)?

3. Is the intervention offered acceptable to the participants and were they able to complete the assessments and intervention without negative consequences?

Two service users were provided with trauma-focused interventions following referral from EIPS. This paper reports the assessment, intervention and outcomes for two cases involving a modular intervention for service users affected by both psychotic and traumatic stress symptoms. 
TFCBT and EMDR for young people with trauma and psychosis

\section{Methods}

\section{Assessment \& Measures}

Both service users completed the standard assessment process within the LTSS. The service uses a standard set of measures. These include:

- Clinician Administered PTSD Scale for DSM-IV (CAPS-4). The CAPS-4 is a 30-item structured interview that can be used to indicate the presence of PTSD according to the DSM-IV definition. Questions also explore the onset and duration of symptoms, subjective distress, impact of symptoms on social and occupational functioning, overall PTSD severity, and dissociation (depersonalization and derealization). Reliability and validity for the original CAPS are high (Hovens et.al., 1994; Blake et.al., 1995; Foa and Tolin, 2000; Mueser et.al., 2001).

- Beck Depression Inventory Second Edition (BDI-II). The BDI-II is a 21-item self-report questionnaire for measuring depression in people age 13 years and above. It was created using the criteria for depression in the Diagnostic and Statistical Manual of Mental Disorders - Fourth Edition (DSM-IV, 1994). Scores range between minimal and severe depression. The BDI-II has high reliability and validity (Beck, Steer and Brown, 1996).

- Beck Anxiety Inventory (BAI - 1993 edition); The BAl is a 21 item scale that measures the severity of anxiety in adults and adolescent. The BAI also has high reliability and validity (Beck and Steer, 1993).

- The Basic Emotion Scale (BES). This scale is designed to assess the experience of a range of emotions derived from the five basics: sadness, anxiety, anger, disgust and happiness (Power, 2006).

- Regulation of Emotions Questionnaire (REQ). This assessment is designed to assess individual differences in the use of strategies to manage difficult or painful emotions. Four 
TFCBT and EMDR for young people with trauma and psychosis

types of emotion regulation strategies are identified: a) Internal - functional; b) Internal dysfunctional; c) External - functional; d) External - dysfunctional. (Power \& Fyvie, 2013)

- Post Traumatic Cognitions Inventory (PTCI). This questionnaire explores thoughts about traumatic events an individual has experienced. The PTCl is a scale on which respondents' rate 33 items on a Likert-type scale ranging from 1 (totally disagree) to 7 (totally agree). Scale scores are formed for three subscales (negative cognitions about the self, negative cognitions about the world, and self-blame), which show a high degree of inter-correlation (rs $=.57-.75)$. Internal consistency appears sound for the three subscales and test-retest reliability for a 1-week interval ranged from .75 to .89 and for a 3-week interval ranged from .80 to .86 for the three subscales. Convergent validity with two other scales that measure trauma-related cognitions is acceptable, as is the ability of the PTCl to differentiate individuals with and without PTSD (sensitivity $=.78$, specificity $=.93$; Foa et al., 1999).

- Dissociative Experiences Scale II (DES-II). The DES-II is a 28-item measure of dissociative symptoms. Each symptom is rated on a scale of $0-100 \%$ of the time; the totals are then added and divided by 28 to get a mean score. Mean scores below 30 are considered indicative of a low level of dissociations and above 30 are considered indicative of a high level of dissociations. The DES has been found to have good reliability and validity (Bernstein and Putnam, 1986).

- Session Rating Scale (SRS) (Johnson et al, 2003). The SRS explores a service user's experience of therapy across 4 factors: relationship; goals and topics; approach and method; and overall satisfaction. This scale was completed by the service user at every session (including all the assessment sessions). Data from this scale offered consistent feedback in regard to the acceptability of the LTSS modular approach.

- The Psychotic Symptom Rating Scales (PSYRATS) (Drake et al 2007) is an instrument designed to quantify the severity of delusions and hallucinations and is typically used in research 
TFCBT and EMDR for young people with trauma and psychosis

studies and clinical settings focusing on people with psychosis and schizophrenia. It is comprised of the auditory hallucinations (AHS) and delusions subscales (DS).

- The Choice Questionnaire (Greenwood et al, 2010) has been developed by asking the opinions of people who have used Cognitive Behaviour Therapy (CBT) to help with their unusual distressing experiences. It supports the identification of treatment goals in CBT.

\section{Pharmacological Assessment and Treatment}

Both service users were offered and both accepted assessment by the service pharmacist with a view to medicines optimisation within the context of trauma-focused psychological intervention. Both service users were offered and took Prazosin to support sleep and manage nightmares (Raskind et al, 2013). Dosage was in line with standard protocols and the service users remained on this regime during the period of active intervention. Their pharmacological regime in relation to psychosis remained unaltered and consistent with treatment as usual.

\section{Intervention}

The LTSS used the following six modules: Module 1: Introduction, Assessment and Formulation (Ehlers and Clark (2000) model, EMDR case conceptualisation in supervision-(van den Berg et al., 2013), Module 2: Pharmacological Treatment (including prazosin), Module 3: Safety, Stabilisation, Building Resources, Behavioural Activation and Education, Module 4: Sleep and Nightmare Management - using Imagery rehearsal therapy (IRT)(Krakow \& Zadra, 2010), Module 5: Focussing on the Trauma - TF-CBT and/or EMDR (three methods approach described in van den Berg et al., 2013) and Module 6: Post trauma growth, relapse prevention and preparing for the future.

To outline the Three Methods Approach: the first method involves identifying difficult life events on a timeline before identify the worst memories that connect with current symptoms. Consequently the therapist would utilise the standard EMDR protocol. The Second Method approach is used to identify memories that underlie the dysfunctional core beliefs or intermediate assumptions of the client that continue to influence their thoughts and behaviours. Starting point in the Second 
TFCBT and EMDR for young people with trauma and psychosis

Method are the negative dysfunctional core beliefs that are connected with the client's problems. Target images of experiences that have led to the formation of these negative beliefs about self, others, and the world (the so called "evidence") are identified. These pieces of evidence are then rated in a hierarchy (from strong to weak "proof") and reprocessed via standard EMDR protocol to obtain a deactivation of the negative schemas or a decrease of their credibility to the client. The third method would be to target any unrealistic and fearful expectations or negative imagery that is associated with the current symptoms.

Following collaborative formulation of service user difficulty, safety and stabilisation interventions (Modules $1-4$ ) were offered in line with the service model.

Intervention was offered predominantly on a weekly basis although there were a few slightly longer gaps due to public holidays. The therapist offering the sessions was closely supervised (on a weekly basis) by a highly experienced therapist (with experience of TF-CBT and EMDR with clients with and without psychosis).

\section{Case Study 1: Sarah - CBT-T and EMDR first method}

\section{Presenting Problem (Both Service Users details have been anonymised)}

Sarah was 20 years old and lived with her parents. She had been bullied throughout school life. Sarah experienced a sexual assault by a family member at age 17 . She reported that she started hearing voices when she was 19 (triggered off by disclosure of the assault). Sarah heard a critical and commanding male voice which she linked to the abuser. She experienced flashbacks of the trauma, nightmares, poor sleep, thoughts of self-harm and suicide, current self-harm, rumination about seeking revenge and a mixture of emotions which included anger, shame and fear. 
TFCBT and EMDR for young people with trauma and psychosis

\section{Assessment and Measures}

Sarah completed the standard set of measures and initial scores on these indicated the presence of significant symptoms of post-traumatic stress and anxiety. Her scores on the DES-II did not indicate high levels and the need to adapt therapy to manage dissociation was not indicated. In addition, the BES and REQ showed higher scores on negative emotions and dysfunctional emotional selfregulation. Table 1 summarises all the measures at the three assessment points.

TABLE 1 HERE

\section{Intervention}

Sarah had previously made links between her sexual trauma and her experience of hearing voices and reported that she needed to work on this trauma so that she could move on. Her main goals for therapy were to improve her mood and reduce how much the voice was troubling her (although ideally she wanted to get rid of the voice all together). Sarah also wanted to stop self-harming and wanted to get back to her old job as a support worker. A collaborative formulation was developed collaboratively with Sarah, based on the Ehlers and Clark model (2000). Module 3 involved working on building resources such as different ways to cope with the voice, emotional regulation techniques and asserting her needs to others. Common responses to sexual trauma were discussed. Imagery Rehearsal Therapy (IRT) was used in module 4 to help Sarah to rescript nightmares that were strongly linked to the traumatic memory. Module 5 helped Sarah to develop a safe place (imagery work) as well as resource development (EMDR protocol). EMDR using eye movements was used to help Sarah to process the sexual assault (first method approach; Van den Berg et al, 2013).

Reliving work was also facilitated. This was completed, at Sarah's request, to assist her to process a particular difficult aspect of her trauma (see protocol by Foa, Hembree, \& Rothbaum, 2007). The final module looked at helping Sarah to continue to repair her reputation (to help reduce feelings of shame). Sarah identified an interest in being involved in service user groups, staff training and has talked about being able to tell her recovery story to help others. 
TFCBT and EMDR for young people with trauma and psychosis

Sarah attended a total of 33 sessions plus one follow up session 6 months after ending therapy.

\section{Outcomes}

\section{Measures}

Sarah's scores on the measures of distress reduced from baseline to the end of therapy. Symptom reduction measures showed a reduction in symptoms of post-traumatic stress, depression and anxiety. Also, improvements were found on the Choice measure. These improvements continued at 6-month follow-up and in addition, her PSYRATS score showed larger improvements at 6month follow-up compared to post-therapy. In addition, the CAPS score was asymptomatic.

The BES and REQ also showed a reduction in negative emotions and dysfunctional emotional strategies. An increase was found in functional emotional strategies and also happiness. This was maintained or improved at 6-month follow-up (see Figures 1 to 3).

The session rating scale showed a slightly lower score for the first session then the highest possible score for all future sessions. This was explored with Sarah and she reported that she was expecting to have to talk about her trauma during the first session (which didn't occur). Overall, these scores demonstrate good engagement.

FIGURES 1 TO 3 HERE

\section{Overall outcomes}

Sarah had managed to improve the quality of her life significantly (having returned to work, playing sports, engaging in an active social life, planning for the future) and reported being more able to cope with her voices and the memories of the trauma. She talked about the usefulness of having her experiences validated and of understanding some common experiences during and after sexual abuse and would have liked to help others to go through this process. After therapy had finished Sarah told her case manager that she had not been taking her antipsychotics for the past year. Sarah has recently been discharged back to her GP. 
TFCBT and EMDR for young people with trauma and psychosis

Table 1 HERE

Figures 1, 2, 3 HERE

\section{Case Study 2: Katy - CBT- T and EMDR second and third methods}

\section{Presenting Problem}

Katy was 17 years old and lived with her parents. She came into the service after being in different adolescent inpatient units. She reported that she heard many different critical voices and saw visions that others could not see (which started when at school). She had history of self-harm and suicide attempts. Katy talked about being bullied throughout school and experienced trauma while on the adolescent units. She experienced flashbacks, dissociation/zoning out, extremely poor sleep, low mood, shame and anger.

\section{Assessment and Measures}

Katy also completed the standard assessment. Her post-traumatic stress symptoms were moderate but she displayed significant anxiety and depression. Her scores on the DES-II did not indicate high levels and the need to adapt therapy to manage dissociation was not indicated. Her BES and REQ scores were also characterised by negative emotions and dysfunctional emotional coping strategies.

\section{Intervention}

Katy talked about multiple traumas and her formulation work identified that her voices and hallucinations were indirectly linked to these traumas (via her core beliefs). Katy also reported strong imagery of future catastrophe/flash-forwards (e.g. attempting to take her own life again). The use of the EMDR case conceptualisation helped to point to the use of the $2^{\text {nd }}$ and $3^{\text {rd }}$ EMDR methods (Van den Berg et al, 2013). Katy's goals for therapy changed throughout and included focusing on sleep and nightmares, developing self-esteem, living with the voices and hallucinations and dealing with traumatic memories. 
TFCBT and EMDR for young people with trauma and psychosis

Katy had already worked on emotional regulation techniques while on an inpatient unit so these techniques were revised (she reported the most useful was mindfulness). Some time was spent working on Katy's interpersonal effectiveness skills and communicating her needs to her parents. The therapist also explored different ways to cope with the voices and visual hallucinations. IRT was used to help Katy to rescript nightmares that were linked to her hallucinations and traumatic memories. Module 5 consisted of using PE to process her two most distressing memories (one was an unwanted sexual experience and the other was of being restrained while on an inpatient unit after trying to end her own life). EMDR second method (using hand pulsars for the bilateral stimulation) was used to process memories connected to Katy's core beliefs (I am weak, I am a disappointment) which were linked to her unusual experiences and other current difficulties. EMDR third method was used to process flash-forwards. The final module focused on helping Katy to keep building up her more adaptive beliefs that emerged during therapy (I am stronger than I thought, I am good enough).

Katy was seen for a total of 32 sessions.

\section{Outcomes}

\section{Measures}

Katy's scores on the measures show improvements in scores for all questionnaires apart from the DES-II and only a very small improvement on the PSYRATS voices questionnaire. The most striking improvements were on the IES-R, BDI, BAI and WSAS showing a reduction in post-traumatic stress, depression, anxiety symptoms and an improvement in daily functioning. At 6 month follow up, her CAPs score was below the clinical cut-off for clinically significant symptoms of PTSD.

On the BES, sustained improvements were found with reducing scores on the subscales of Fear and Sadness, and a sustained increase in happiness. Post-therapy, the scores on Disgust and Anger had reduced but had increased almost to pre-therapy levels within 6 months. On the REQ, maintained 
TFCBT and EMDR for young people with trauma and psychosis

increases in both external and internal functioning were found and sustained reductions in the respective dysfunction scores from pre-therapy to six-month follow-up.

Katy's scores on the session rating scale were similar to Sarah's with slightly lower than maximum score at first appointment but then the maximum score for the remaining appointments.

Table 2 HERE

Figures 4, 5, 6 HERE

\section{Overall outcomes}

Katy has reported that she now experiences much less distress from her trauma/aversive life event memories and is starting to build up more positive beliefs about herself. She stated that the trauma therapy helped her to change how she feels about her voices and visual hallucinations and feels she is more able to accept them and to carry on with her life. She had not made any attempts at taking her own life in the past year and had reported that she felt more confident about her ability to cope with life. Katy reported that her relationships in her family had improved and Katy said she felt more hopeful for the future. Katy had plans to go to university in the future

\section{Summary of intervention and outcomes for both service users}

Within Module 5 both clients were given the choice of starting with TF-CBT, which included prolonged exposure (Foa et al, 2007) or EMDR (Shapiro 2001). In the present study, a significantly larger amount of preparation work (modules 1-4) was completed. They were both offered and took up a combination of TF-CBT and EMDR, as guided by a collaborative framework including a shared formulation.

Overall, the two service users demonstrated clear benefits from engaging in trauma-focused psychological intervention. This was evidenced by their improvement in functioning as well as clinically significant reductions in the assessment measures used within the service. 
TFCBT and EMDR for young people with trauma and psychosis

\section{Discussion}

The aims of this case series were to investigate how feasible, acceptable and useful TF-CBT and EMDR are for young people with experiences of trauma and psychosis, within a phasic treatment approach.

For both Sarah and Katy, positive outcomes were achieved by the end of therapy and also at 6month follow-up. The outcome measures have demonstrated clinically significant reductions on measures of mood and post-traumatic stress reducing to below caseness thresholds. The use of the BES and REQ indicated improvements in positive emotions and coping strategies for both service users. However, for one of the case studies (Katy) the scores on anger and disgust increased, though a number of life events and historic relational difficulties may well account for this. Both service users were positive about their therapy experience and felt it had helped them to move on. The qualitative feedback included: "I would like to tell others that if they are thinking about trying this therapy, they should stick with it. Even though it is a lot of commitment and hard work, it pays off."

The findings of this study indicated that both TF-CBT and EMDR have been useful for both clients (used in an integrated way, incorporating other approaches to psychosis such as ways of managing distressing voices). The phasic approach felt containing for both the client and the therapist and allowed time to build up the clients' understanding and coping skills so that they have felt more able to face their traumatic experiences.

The aims of the study were met in that both service users were able to engage with the intervention across the modular approach (aims 1 and 2). The intervention offered was acceptable (aim 3) as evidenced by their session rating scale and completion of all the assessment measures.

\section{Links with previous research}

The results from this study show some similarities to the T-TIP and RCT studies done in Holland (Van den Berg et al, 2015) in that trauma-focused approaches seem to be feasible and acceptable for 
TFCBT and EMDR for young people with trauma and psychosis

some service users with psychosis. In comparison with the Dutch studies, a significantly larger amount of preparation work (modules 1-4) was done in these two cases. They were both offered and took up a combination of TF-CBT and EMDR and neither had a diagnosis of co-morbid PTSD. On reflection, the authors of this paper would aim to adopt this approach using less sessions for future service-users.

The results from this discussion point to a need to focus on treating trauma in psychosis for clients who feel ready to do so.

\section{Limitations}

There are some limitations to this study. The approaches were only trialled with two participants. However, Katy and Sarah both have complicated life stories and a wide range of difficulties which do seem to match the majority of other early intervention service clients. The therapist administered most of the outcome measures; the scores are therefore more at risk of being biased by the therapeutic relationship and/or the need to please the therapist or the therapist scoring the questionnaires to fit in with the hoped for findings. There was no use of a control group so assumptions cannot be made that the changes seen were down to the therapy alone. Both Katy and Sarah had input from their case managers (who had been trained in psychosocial interventions) and so it is likely that their sessions contributed to the positive outcomes. Sarah and Katy were seen for a year each; this was necessary to allow time to explore the usefulness and impact of the different approaches/techniques. It was also felt that the length of therapy enabled the development of strong and trusting relationship with the therapist so that the clients felt able to talk about the very worst parts of their traumatic experiences. NICE guidelines currently recommend that service users are offered between 16 and 20 sessions of CBT (NICE, 2005).

It is worth noting the setting in which this therapy took place. The clients were seen at a trauma service clinic which was set up for this purpose (calm, quiet, welcoming waiting area, high level of input and support from admin staff at reception). Sarah and Katy both commented on how helpful it 
TFCBT and EMDR for young people with trauma and psychosis

was to come for therapy there and that they felt more able to work on their aversive or traumatic experiences than in other settings (EIS home visits or busy community clinics). The therapist received intensive weekly one to one supervision as well as weekly peer support discussions. This structure supports therapist efficacy as well as promoting a supportive culture to prevent vicarious traumatisation and burnout. These factors would be difficult to replicate in every day early intervention or community mental health services and so this limits the degree to which we can extrapolate from these findings. This raises questions about ways of working in early intervention services and the possibility of moving away from the culture of mainly offering home visits (with the rationale of reducing distress and stigma linked to being seen in mental health clinics).

A further issue relates to the extensiveness of therapy. There were five modules plus the use of medication to reduce nightmares. In addition, input from the EIP service was not recorded in detail for the purposes of the current study. Some components might be shortened or removed with no detrimental effect and this cannot be determined from the present study. Optimising medication would have potentially impacted on service user wellbeing and as such also be considered.

\section{Future research}

Future research could involve a larger feasibility study on the use of the TF-CBT and/or EMDR for young people with trauma and psychosis (in early intervention services), within the modular approach. Clearly, a more extensive trial would be required in which outcomes could be compared with patients receiving treatment as usual in EIP services. Following on from this, a multi-centre randomised trial would help to contribute to the evidence base for these approaches and could potentially shape future guidance on therapy for people with experiences of trauma and psychosis. Qualitative studies on service users' experiences of receiving these approaches, with specific focus on EMDR given its infancy in the psychosis area, would provide more detailed and meaningful information on what aspects of the therapy have an impact and which are the most useful. This 
TFCBT and EMDR for young people with trauma and psychosis

would build on previous studies on service users' experiences of trauma-focused therapy such as Lowe and Murray (2014).

\section{Conclusion}

In summary, this case series and the small number of other mentioned studies are starting to build up evidence of significant positive outcomes for service users with trauma and psychosis, receiving trauma-focused approaches. The use of EMDR with psychosis is still very much in its infancy and more research needs to be done to explore its feasibility, acceptability and effectiveness as well as examining the underlying mechanisms involved in any progress made in therapy. The culture of professionals feeling worried about working with trauma in psychosis is starting to shift but still has a long way to go. The use of phasic/modular approaches also warrants further exploration and could provide a useful framework to help service users to feel ready to face the aversive or traumatic life events that have contributed to their current difficulties and distress. 
TFCBT and EMDR for young people with trauma and psychosis

\section{References}

Baird, K., Kracen, A. C. (2006) Vicarious traumatization and secondary traumatic stress: A research synthesis. Counselling Psychology Quarterly, 19(2): 181-188

Beck, A.T., Steer, R.A., Brown, G.K. (1996) Manual for the Beck Depression Inventory ${ }^{\circledast}\left(2^{\text {nd }} E d.\right)$, San Antonio, TX: The Psychological Corporation.

Beck, A.T., Steer, R.A., Brown, G.K. (1993) Manual for the Beck Anxiety Inventory ${ }^{\circledR}$ San Antonio, TX: The Psychological Corporation.

Bentall, R. P., Wickham, S., Shevlin, M. \& Varese, F (2012). Do specific early-life adversities lead to specific symptoms of psychosis? Schizophrenia Bulletin, 38, 734-740.

Carlson, E.B. and Putnam, F.W. (1993) An Update on the Dissociative Experiences Scale. Dissociation $1,1$.

Cloitre, M., Courtois, C. A., Charuvastra, A., Carapezza, R., Stolbach, B. C., Green, B. L. (2011). Treatment of Complex PTSD: Results of the ISTSS Expert Clinician Survey on Best Practice. Journal of Traumatic Stress, Vol. 24, No. 6, December 2011, pp. 615-627

Creamer, M., Bell, R., Faillan, S. (2003) Psychometric properties of the Impact of Event Scale Revised. Behavioural Research and Therapy, 4, 1489-1496.

De Bont, M., Van den Berg, D. P., \& Van der Vleugel, B. M. (2013). Multi-site single blind clinical study to compare the effects of prolonged exposure, eye movement desensitization and reprocessing and waiting list on patients with a current diagnosis of psychosis and co morbid posttraumatic stress disorder: study protocol for the randomized controlled trial Treating Trauma in Psychosis (T-TIP). Trials, 14, 151. http://dx.doi.org/10.1186/1745-6215-14-151 
TFCBT and EMDR for young people with trauma and psychosis

De Bont, P. A., Van Minnen, A., De Jongh, A. (2013) Treating PTSD in patients with psychosis: a within-group controlled feasibility study examining the efficacy and safety of evidence-based PE and EMDR protocols. Behaviour Therapy, 44(4):717-730.

Ehlers, A and Clark, D.M (2000) A cognitive model of posttraumatic stress disorder. Behaviour Research and Therapy, 38, 319-345

Foa, E. B., Hembree, E. A., Rothbaum, B. O. (2007). Prolonged exposure therapy for PTSD: Emotional processing of traumatic experiences: Therapist guide. Oxford, England: Oxford University Press.

Frueh, B. C., Grubaugh, A. L., Cusack, K. J., Kimble, M. O., Elhai, J. D., \& Knapp, R, G. (2009) Exposurebased cognitive-behavioral treatment of PTSD in adults with schizophrenia or schizoaffective disorder: a pilot study. Journal of Anxiety Disorders, 23(5):665-675.

Greenwood K. E., Sweeney A., Williams S., Garety P., Kuipers E., Scott J., Peters E. (2010). CHoice

of Outcome In Cbt for psychosEs (CHOICE): The Development of a New Service-User led

Outcome Measure of CBT for Psychosis. Schizophrenia Bulletin 36(1) 126-135.

Howgego, I. M., Owen, C., Meldrum, L., Yellowlees, P., Dark, F., \& Parslow, R. (2005). Posttraumatic stress disorder: An exploratory study examining rates of trauma and PTSD and its effects on client outcomes in community mental health. BMC Psychiatry, 5(21).

Johnson, L. D., Duncan, B. L., Miller, S. D, Spark, J. A., Claud, D. A., Reynolds, L. R., Brown, J. (2003) The Session Rating Scale: Preliminary Psychometric Properties of a "Working" Alliance Measure Journal of Brief Therapy Vol 3 Number 1

Krakow, B. and Zadra, A. (2010) Imagery Rehearsal Therapy: Principles and Practice. Elsevier Sleep Medicine Clinics, 5, 289-298.

Lowe, C., \& Murray, C. (2014). Adult Service-Users' Experiences of Trauma-Focused Cognitive Behavioural Therapy. Journal of Contemporary Psychotherapy, 44 (2), DOI 10.1007/s10879-014$9272-1$ 
TFCBT and EMDR for young people with trauma and psychosis

Morrison, A. P., Frame, L., \& Larkin, W. (2003). Relationships between trauma and psychosis: A review and integration. British Journal of Clinical Psychology, 42, 331-353.

National Institute for Health and Care Excellence (2005) Post-traumatic stress disorder: management. Clinical guideline [CG26].

National Institute for Health and Care Excellence (2014) Psychosis and schizophrenia in adults: prevention and management. Clinical guideline [CG178]

NHS England, the National Collaborating Centre for Mental Health and the National Institute for Health and Care Excellence (2016) Implementing the Early Intervention in Psychosis Access and Waiting Time Standard: Guidance. NHS England Publications Gateway Reference 04294

Phillips, K.F.V. \& Power, M.J. (2007) A New Self-Report Measure of Emotion Regulation in Adolescents: The Regulation of Emotions Questionnaire. Clinical Psychology and Psychotherapy, 14, $145-156$.

Power, M. J. (2006) The structure of emotion: an empirical comparison of six models. Cognition and Emotion, 20, 694-713.

Power, M., A Fyvie, C. (2013) The role of emotions in PTSD: two preliminary studies. Behavioural \& Cognitive Psychotherapy, 41, $162-172$

Raskind, M.A., Peterson, K., Williams, T., Hoff, D.J., Hart, K., Holmes, H., Homas, D., Hill, J., Daniels, C., Calohan, J., Millard, S.P., Rohde, K., O'Connell, J., Pritz, I D., Feiszli, K., Petrie, E.C., Gross, C., Mayer, C.L., Freed, M.C., Engel, C., Peskind, E.R. (2013) A trial of prazosin for combat trauma PTSD with nightmares in active-duty soldiers returned from Iraq and Afghanistan. American Journal of Psychiatry, 1003-10.

Shapiro, F. (2001). Eye movement desensitization and reprocessing: Basic principles, protocols and procedures (2nd edition). New York: Guilford Press. 
TFCBT and EMDR for young people with trauma and psychosis

Sitko, K. Bentall, RP, Shevlin, M. O'Sullivan, N. \& Sellwood W. (2014) Associations between specific psychotic symptoms and specific childhood adversities are mediated by attachment styles: An analysis of the National Comorbidity Survey. Psychiatry Research: 217, 202-209

Thomas, N., Hayward, M., Peters, E., van der Gaag, M., Bentall, R. P., Jenner, J., Strauss, C., Sommer, I. E., Johns, L. C, Varese, F., Garcia-Montes, J. M., Waters, F., Dodgson, G., McCarthy-Jones, S. (2014) Psychological Therapies for Auditory Hallucinations (Voices): Current Status and key Directions for Future Research. Schizophrenia Bulletin, 40 (4), s202-s212. Doi:10.1093/schbul/sbu037.

Van den Berg, D. P., Van der Vleugel, D. M., Staring, A. B., De Bont, P. A., \& De Jongh, A. (2015) EMDR in Psychosis: Guidelines for conceptualisation and treatment. Journal of EMDR Practice and Research, 7 (4), 208-224.

Van den Berg, D. P., Van der Gaag, M. (2012) Treating trauma in psychosis with EMDR: a pilot study. Journal of Behavioural Therapy and Experimental Psychiatry. 2012; 43(1):664-671.

Van Den Berg, D. P. M., Van der Vleugel, B. M., Staring, A. B. P., De Bont, P. A. J., De Jongh, A. (2013). EMDR in Psychosis: Guidelines for Conceptualization and Treatment. Journal of EMDR Practice and Research, 7 (4), 208-224. http://dx.doi.org/10.1891/1933-3196.7.4.208

Varese, F., Smeets, F., Drukker, M., Lieverse, R., Lataster, T., Viechtbauer, W., Read, J., van Os, J., \& Bentall, R. (2012). Childhood Adversities Increase the Risk of Psychosis: A Meta-analysis of PatientControl, Prospective- and Cross-sectional Cohort Studies. Schizophrenia Bulletin, 38 (4), 661-671. doi:10.1093/schbul/sbs0505.

Watts, B. V., Schnurr, P. P., Mayo, L., Young-Xu, Y., Weeks, W. B., \& Friedman, M. J. (2013) Metaanalysis of the efficacy of treatments for posttraumatic stress disorder. Journal of Clinical Psychiatry, 74(6), e541-e550. doi:10.4088/JCP.12r08225. 
Table 1: Sarah's pre-therapy, post-therapy and 6 month follow-up scores

\begin{tabular}{|c|c|c|c|c|c|c|}
\hline Measure & \multicolumn{2}{|c|}{ Pre-therapy score } & \multicolumn{2}{|c|}{ Post-therapy score } & \multicolumn{2}{|c|}{$\begin{array}{c}6 \text { month follow-up } \\
\text { score }\end{array}$} \\
\hline $\begin{array}{c}\text { Impact of Events } \\
\text { Scale Revised (IES-R) }\end{array}$ & \multicolumn{2}{|l|}{71} & \multicolumn{2}{|c|}{25} & \multicolumn{2}{|l|}{8} \\
\hline $\begin{array}{l}\text { Choice (CQ-High } \\
\text { score=better } \\
\text { recovery) }\end{array}$ & \multicolumn{2}{|l|}{57} & \multicolumn{2}{|c|}{82} & \multicolumn{2}{|c|}{92} \\
\hline PSYRATS & \multicolumn{2}{|c|}{$V=33$} & \multicolumn{2}{|c|}{$V=29$} & \multicolumn{2}{|c|}{$B=0$} \\
\hline $\begin{array}{l}\text { Work and Social } \\
\text { Adjustment Scale } \\
\text { (WSAS) }\end{array}$ & \multicolumn{2}{|l|}{19} & \multicolumn{2}{|c|}{7} & \multicolumn{2}{|c|}{0} \\
\hline \multirow{5}{*}{$\begin{array}{l}\text { Basic Emotions Scale } \\
\text { (BES) (Maximum } \\
\text { score on each domain } \\
\text { scale is 28) }\end{array}$} & Anger & 15 & Anger & 12 & Anger & 4 \\
\hline & Disgust & 18 & Disgust & 8 & Disgust & 4 \\
\hline & Sadness & 14 & Sadness & 4 & Sadness & 4 \\
\hline & Fear & 15 & Fear & 12 & Fear & 4 \\
\hline & Happiness & 17 & Happiness & 25 & Happiness & 26 \\
\hline \multirow{4}{*}{$\begin{array}{l}\text { Regulation of } \\
\text { Emotions } \\
\text { Questionnaire (REQ2) } \\
\text { Scoring is a mean } \\
\text { score from each } \\
\text { scale, maximum score } \\
\text { is } 6\end{array}$} & $\begin{array}{c}\text { Internal } \\
\text { Dysfunction }\end{array}$ & 2.8 & $\begin{array}{c}\text { Internal } \\
\text { Dysfunction }\end{array}$ & 1.8 & $\begin{array}{c}\text { Internal } \\
\text { Dysfunction }\end{array}$ & 1 \\
\hline & $\begin{array}{l}\text { Internal } \\
\text { Function }\end{array}$ & 3.2 & $\begin{array}{l}\text { Internal } \\
\text { Function }\end{array}$ & 2.8 & $\begin{array}{l}\text { Internal } \\
\text { Function }\end{array}$ & 3.8 \\
\hline & $\begin{array}{c}\text { External } \\
\text { Dysfunction }\end{array}$ & 1.6 & $\begin{array}{c}\text { External } \\
\text { Dysfunction }\end{array}$ & 1 & $\begin{array}{c}\text { External } \\
\text { Dysfunction }\end{array}$ & 1 \\
\hline & $\begin{array}{l}\text { External } \\
\text { Function }\end{array}$ & 2.8 & $\begin{array}{l}\text { External } \\
\text { Function }\end{array}$ & 4 & $\begin{array}{l}\text { External } \\
\text { Function }\end{array}$ & 4 \\
\hline $\begin{array}{l}\text { Beck Depression } \\
\text { Inventory (BDI-II) }\end{array}$ & \multicolumn{2}{|l|}{18} & \multicolumn{2}{|c|}{17} & \multicolumn{2}{|c|}{1} \\
\hline $\begin{array}{l}\text { Beck Anxiety } \\
\text { Inventory (BAI) }\end{array}$ & \multicolumn{2}{|l|}{25} & \multicolumn{2}{|c|}{13} & \multicolumn{2}{|c|}{1} \\
\hline $\begin{array}{l}\text { Clinician } \\
\text { Administered PTSD } \\
\text { scale (CAPS) }\end{array}$ & \multicolumn{2}{|c|}{ (severe and extreme) } & \multicolumn{2}{|c|}{$\begin{array}{c}\text { (mild } \\
\text { PTSD/subthreshold) }\end{array}$} & \multicolumn{2}{|c|}{$\begin{array}{c}4 \\
\text { (asymptomatic/few } \\
\text { symptoms) }\end{array}$} \\
\hline $\begin{array}{l}\text { Dissociative } \\
\text { Experiences Scale }\end{array}$ & \multicolumn{2}{|c|}{21} & \multicolumn{2}{|c|}{11} & \multicolumn{2}{|c|}{4.6} \\
\hline
\end{tabular}


\begin{tabular}{|l|l|l|}
\hline & & \\
\hline
\end{tabular} 
Table 2: Katy's pre-therapy, post-therapy and 6 month follow-up scores

\begin{tabular}{|c|c|c|c|c|c|c|}
\hline Measure & \multicolumn{2}{|c|}{ Pre-therapy score } & \multicolumn{2}{|c|}{ Post-therapy score } & \multicolumn{2}{|c|}{$\begin{array}{c}6 \text { month follow-up } \\
\text { score }\end{array}$} \\
\hline $\begin{array}{l}\text { Impact of Events } \\
\text { Scale Revised (IES-R) }\end{array}$ & \multicolumn{2}{|c|}{59} & \multicolumn{2}{|c|}{8} & \multicolumn{2}{|c|}{2} \\
\hline $\begin{array}{l}\text { Choice (CQ-High } \\
\text { score=better } \\
\text { recovery) }\end{array}$ & \multicolumn{2}{|c|}{57} & \multicolumn{2}{|c|}{83} & \multicolumn{2}{|c|}{97} \\
\hline PSYRATS & \multicolumn{2}{|c|}{$V=30$} & \multicolumn{2}{|c|}{$V=29$} & \multicolumn{2}{|c|}{$B=5$} \\
\hline $\begin{array}{l}\text { Work and Social } \\
\text { Adjustment } \\
\text { (WSAS) }\end{array}$ & \multicolumn{2}{|c|}{14} & \multicolumn{2}{|c|}{4} & \multicolumn{2}{|c|}{4} \\
\hline \multirow{5}{*}{$\begin{array}{l}\text { Basic Emotions Scale } \\
\text { (BES) }\end{array}$} & Anger & 18 & Anger & 9 & Anger & 13 \\
\hline & Disgust & 21 & Disgust & 7 & Disgust & 12 \\
\hline & Sadness & 10 & Sadness & 7 & Sadness & 7 \\
\hline & Fear & 19 & Fear & 12 & Fear & 10 \\
\hline & Happiness & 14 & Happiness & 23 & Happiness & 24 \\
\hline \multirow{4}{*}{$\begin{array}{l}\text { Regulation of } \\
\text { Emotions } \\
\text { Questionnaire (REQ2) }\end{array}$} & $\begin{array}{c}\text { Internal } \\
\text { Dysfunction }\end{array}$ & 3.8 & $\begin{array}{c}\text { Internal } \\
\text { Dysfunction }\end{array}$ & 2.2 & $\begin{array}{c}\text { Internal } \\
\text { Dysfunction }\end{array}$ & 2.2 \\
\hline & $\begin{array}{l}\text { Internal } \\
\text { Function }\end{array}$ & 2.8 & $\begin{array}{l}\text { Internal } \\
\text { Function }\end{array}$ & 4.2 & $\begin{array}{l}\text { Internal } \\
\text { Function }\end{array}$ & 4 \\
\hline & $\begin{array}{c}\text { External } \\
\text { Dysfunction }\end{array}$ & 2.6 & $\begin{array}{c}\text { External } \\
\text { Dysfunction }\end{array}$ & 1 & $\begin{array}{c}\text { External } \\
\text { Dysfunction }\end{array}$ & 1.2 \\
\hline & $\begin{array}{l}\text { External } \\
\text { Function }\end{array}$ & 2.2 & $\begin{array}{l}\text { External } \\
\text { Function }\end{array}$ & 4 & $\begin{array}{l}\text { External } \\
\text { Function }\end{array}$ & 4.2 \\
\hline $\begin{array}{l}\text { Beck Depression } \\
\text { Inventory (BDI) }\end{array}$ & \multicolumn{2}{|c|}{18} & \multicolumn{2}{|c|}{2} & \multicolumn{2}{|l|}{0} \\
\hline $\begin{array}{l}\text { Beck Anxiety } \\
\text { Inventory (BAI) }\end{array}$ & \multicolumn{2}{|c|}{25} & \multicolumn{2}{|c|}{4} & \multicolumn{2}{|c|}{0} \\
\hline $\begin{array}{l}\text { Clinician } \\
\text { Administered } \quad \text { PTSD } \\
\text { scale (CAPS) }\end{array}$ & \multicolumn{2}{|c|}{$\begin{array}{c}\text { (moderate } \\
\text { PTSD/threshold) }\end{array}$} & \multicolumn{2}{|c|}{$\begin{array}{c}\text { (mild } \\
\text { PTSD/subthreshold) }\end{array}$} & \multicolumn{2}{|c|}{$\begin{array}{c}16 \\
\text { (subthreshold) }\end{array}$} \\
\hline $\begin{array}{l}\text { Dissociative } \\
\text { Experiences Scale }\end{array}$ & \multicolumn{2}{|c|}{21} & \multicolumn{2}{|c|}{23.6} & \multicolumn{2}{|c|}{5.7} \\
\hline
\end{tabular}


\begin{tabular}{|l|l|l|}
\hline & & \\
\hline
\end{tabular} 
Figure 1: Sarah's pre, post and follow-up scores

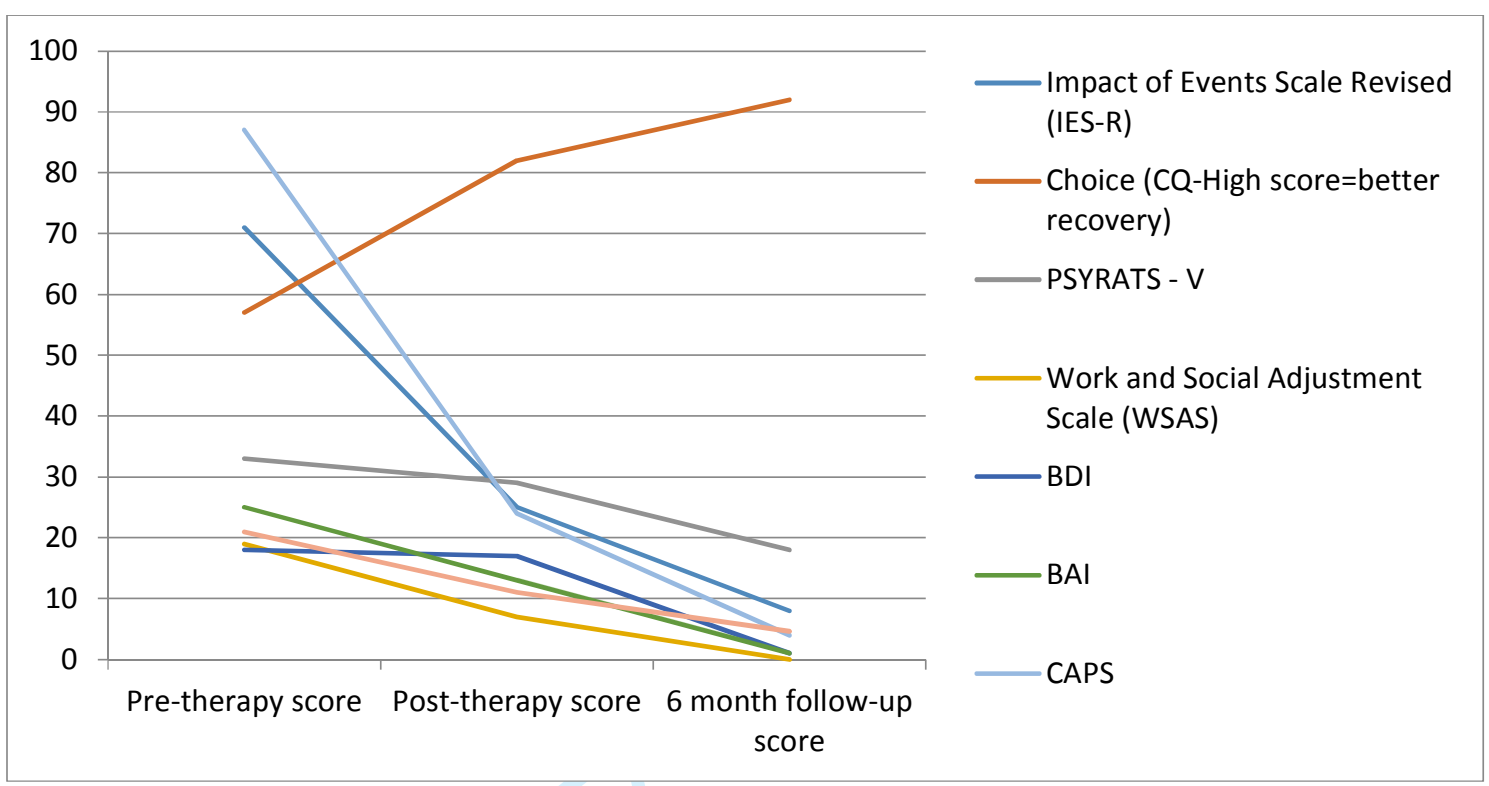


Figure 2: Sarah's pre, post and follow-up scores (Basic Emotions Scale)

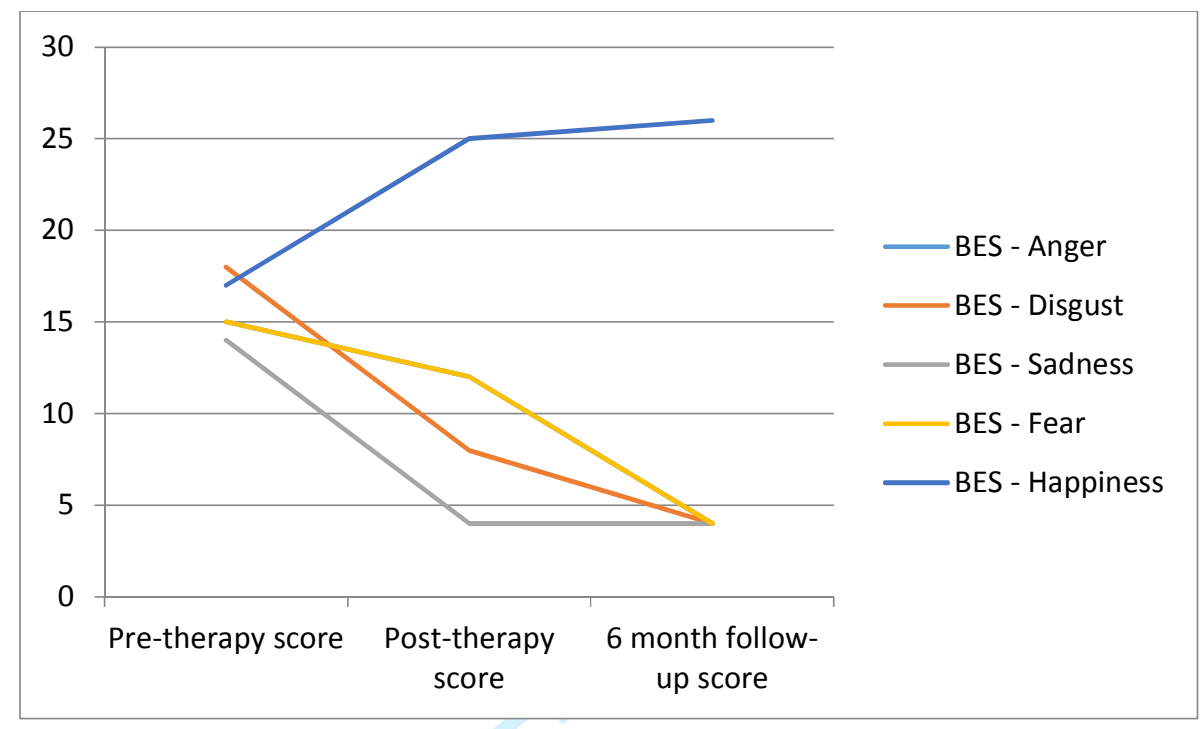


Figure 3: Sarah's pre, post and follow-up scores (Regulation of Emotions Questionnaire)

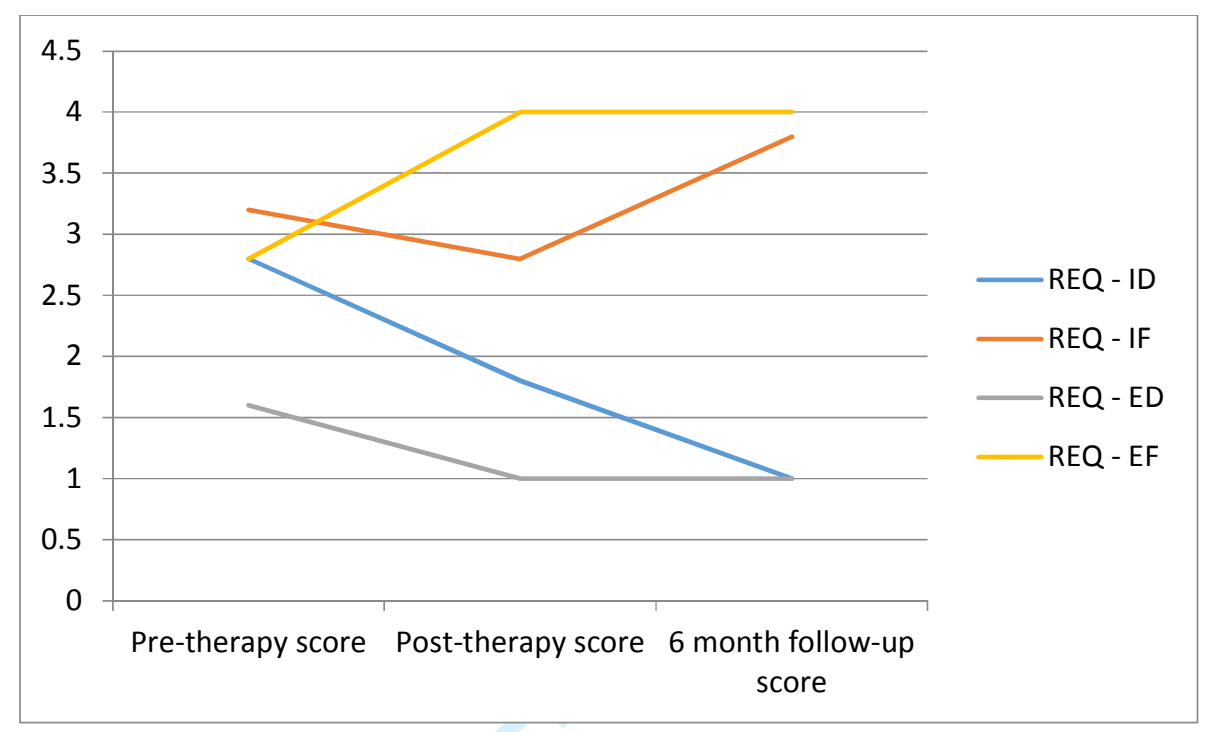


Figure 4: Katy's pre, post and follow-up scores

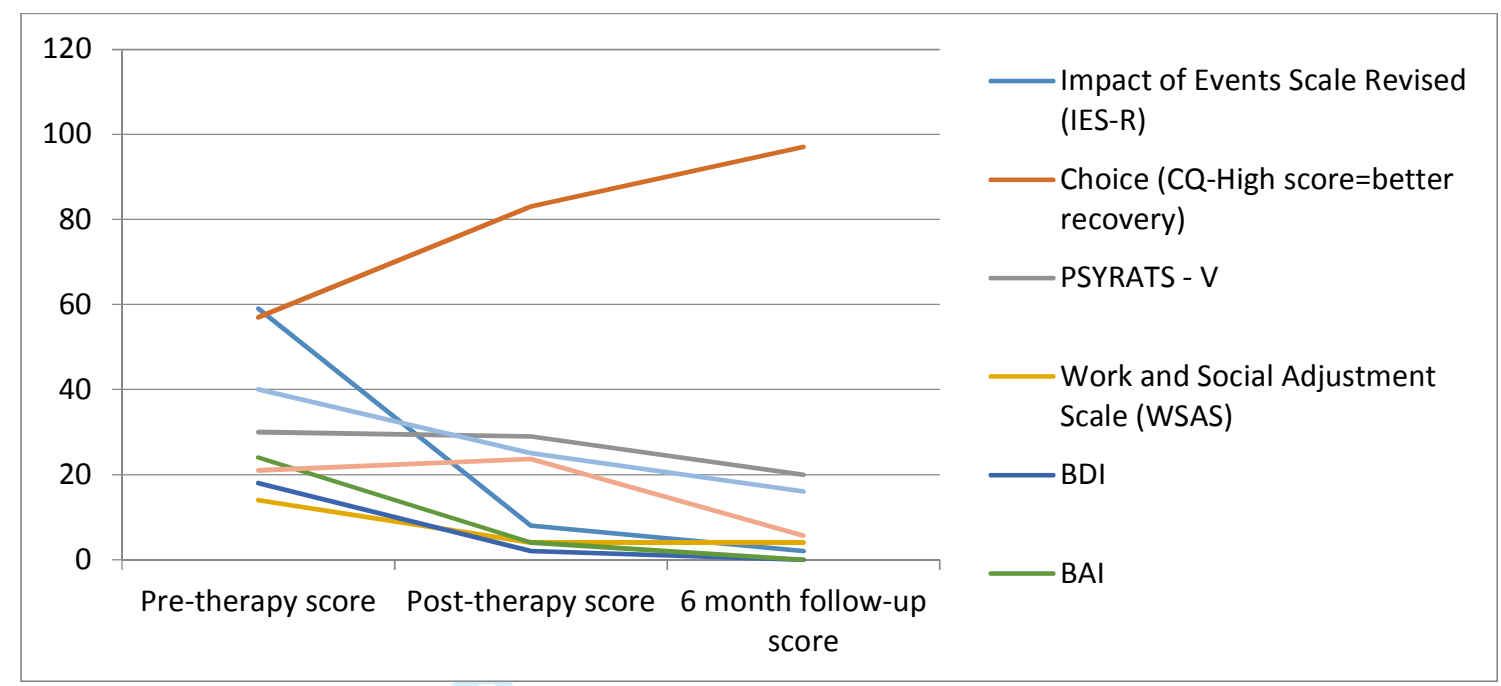


Figure 5: Katy's pre, post and follow-up scores (Basic Emotions Scale)

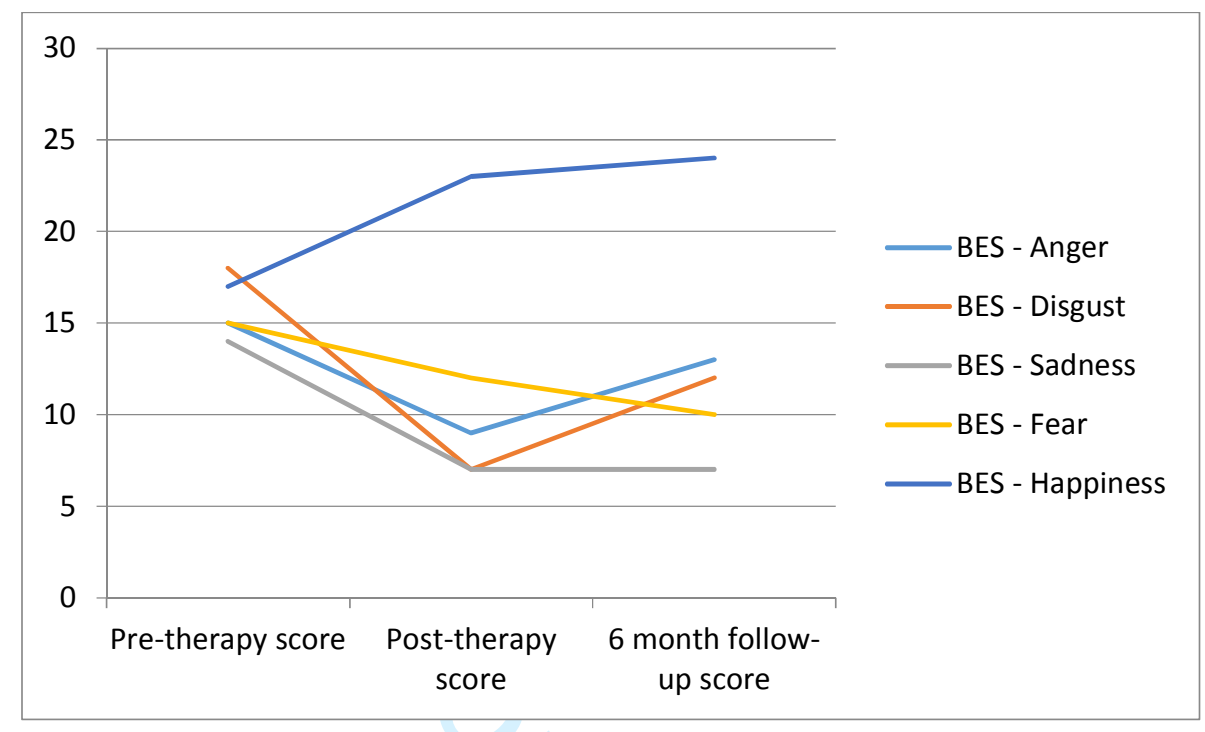


Figure 6: Katy's pre, post and follow-up scores (Regulation of Emotions Questionnaire)

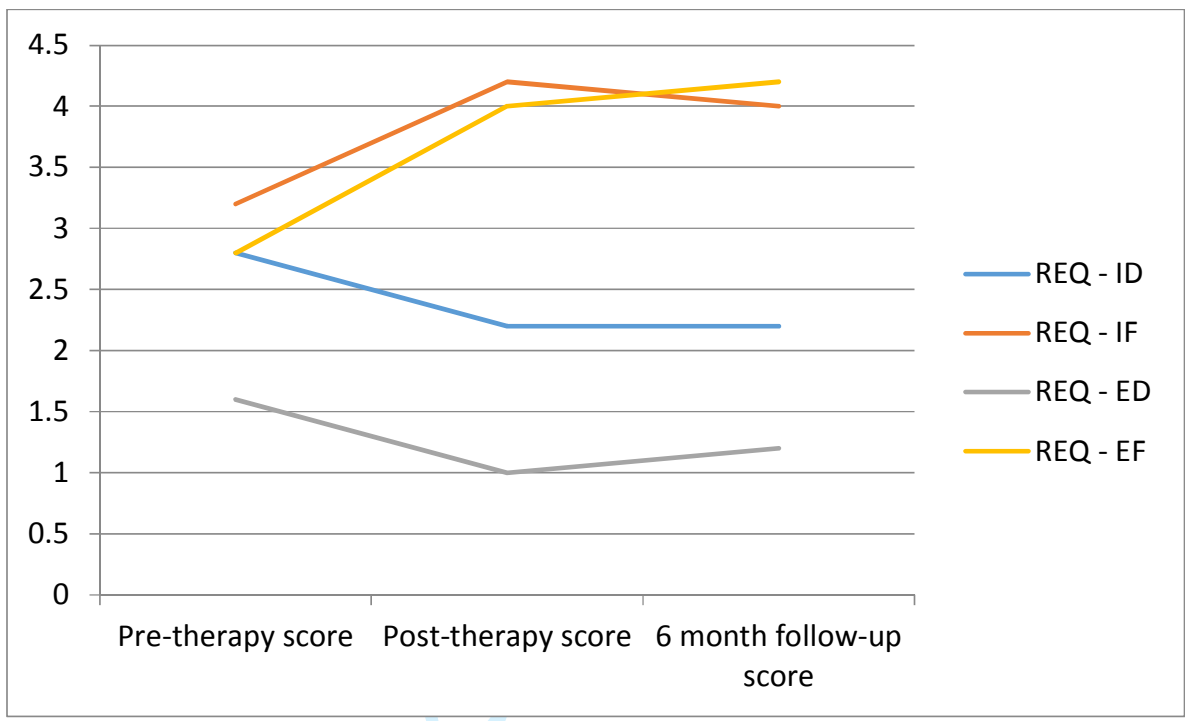

